Old Dominion University

ODU Digital Commons

Physics Faculty Publications

Physics

2009

\title{
Comment on "On the Theory of Nuclear Resonant Forward Scattering of Synchrotron Radiation"
}

Gilbert R. Hoy

Old Dominion University

Jos Odeurs

Follow this and additional works at: https://digitalcommons.odu.edu/physics_fac_pubs

Part of the Condensed Matter Physics Commons

\section{Repository Citation}

Hoy, Gilbert R. and Odeurs, Jos, "Comment on "On the Theory of Nuclear Resonant Forward Scattering of Synchrotron Radiation"'" (2009). Physics Faculty Publications. 200.

https://digitalcommons.odu.edu/physics_fac_pubs/200

\section{Original Publication Citation}

Hoy, G. R., \& Odeurs, J. (2009). Comment on "On the theory of nuclear resonant forward scattering of synchrotron radiation".

Physical Review B, 79(10), 106401. doi:10.1103/PhysRevB.79.106401

This Article is brought to you for free and open access by the Physics at ODU Digital Commons. It has been accepted for inclusion in Physics Faculty Publications by an authorized administrator of ODU Digital Commons. For more information, please contact digitalcommons@odu.edu. 


\title{
Comment on "On the theory of nuclear resonant forward scattering of synchrotron radiation"
}

\author{
Gilbert R. Hoy \\ Department of Physics, Old Dominion University, Norfolk, Virginia 23529-0116, USA \\ Jos Odeurs \\ Instituut voor Kern-en Stralingsfysica, Katholieke Universiteit Leuven, Celestijnenlaan 200 D, B-3001 Leuven, Belgium
}

(Received 21 April 2008; revised manuscript received 1 August 2008; published 20 March 2009)

\begin{abstract}
Recently, in a paper by Kohn and Smirnov, a formula previously derived by Kagan et al. was developed to explain the forward scattering of gamma radiation by a nuclear-resonant sample excited by pulsed synchrotron radiation. Their derivation followed, directly, a procedure developed by Heitler, Harris, and Hoy. Previously, a completely different formula was developed by Hoy et al. to explain the same process. As a result, Kohn and Smirnov discuss the correctness and validity of the two models. In this Comment a detailed numerical comparison of the two theories has also been made. It is shown that their comparison is substantially inaccurate. The two models give essentially the same results. There is some small difference at times long after the synchrotron radiation pulse. If experiments of this type are used to extract nuclear parameters, either model will provide the same results. Either model will fit the experimental data well.
\end{abstract}

DOI: 10.1103/PhysRevB.79.106401

PACS number(s): 76.80.+y, 78.90.+t, 42.25.Bs

\section{INTRODUCTION}

We would like to comment on the recent paper by Kohn and Smirnov. ${ }^{1}$ In their paper Kohn and Smirnov use the methods of Heitler, ${ }^{2}$ Harris, ${ }^{3}$ and $\mathrm{Hoy}^{4}$ to obtain the same solution as already given by Kagan et al. $^{5}$ to describe nuclear-resonant forward scattering of synchrotron radiation. We do not quarrel with their derivation of the so-called semiclassical optical model (SCOM) result. One can say, a priori, that their result is correct since Harris ${ }^{3}$ did exactly the same thing, using the Heitler approach to obtain the semiclassical optical model solution for the radioactive source case that Hamermesh and co-workers ${ }^{6}$ had already obtained. Our concern has to do with the comparison Kohn and Smirnov make of the semiclassical optical model with the coherent-path model (CPM). We will show that the majority of their statements on this subject are not true.

\section{TWO MODELS}

The two models in question are the semiclassical optical model $^{1}$ and the coherent-path model. ${ }^{7}$ To simplify the comparison, we confine our attention to the case where there is just one resonance transition in the resonant sample having frequency $\omega_{j}$.

\section{A. SCOM}

In the SCOM the amplitude for finding a resonant photon of frequency $\omega_{j}$ at the detector, at time $t$ after the synchrotron radiation pulse, and after passing through a sample of thickness $d$ is given by ${ }^{1}$

$$
A_{\mathrm{SCOM}}(\beta, t)=e^{-i \omega_{j} t-(t / 2 \tau)}\left(\frac{1}{\tau}\right)\left[\frac{\beta \tau}{4 t}\right] J_{1}\left(\left[\frac{\beta t}{\tau}\right]^{1 / 2}\right) .
$$

$J_{1}$ is the Bessel function of first order and the nuclearresonant thickness parameter is given by $\beta=N_{0} \sigma_{0} f \eta d$. Here $N_{0}$ is the number of resonant nuclei per $\mathrm{cm}^{3}, \sigma_{0}$ is the maxi- mum cross section evaluated on resonance, $f$ is the recoilfree fraction, $\eta$ is the isotopic enrichment of the sample, and $d$ is the thickness of the sample. The mean lifetime $\tau$ of the first excited nuclear level is $\tau=\frac{\hbar}{\Gamma}$, where $\Gamma$ is the energy full width of the first excited nuclear level.

\section{B. CPM}

The corresponding equation for the CPM (Ref. 7) is

$$
A_{\mathrm{CPM}}(N, t)=e^{-i \omega_{j} t-(t / 2 \tau)}\left[N+\sum_{n=1}^{N-1}\left(\frac{-f \gamma_{R}}{2 \hbar}\right)^{n}\left(\begin{array}{c}
N \\
n+1
\end{array}\right) \frac{t^{n}}{n !}\right] \text {. }
$$

In Eq. (2) $N$ is the number of effective resonant nuclei in the one-dimensional chain representing the sample or equivalently one could say the number of stacked "effective" nuclear resonant planes in the sample. The factor $\gamma_{R}$ is the radiative width of the first excited nuclear level and $\left(\begin{array}{c}N \\ n+1\end{array}\right)$ is a binomial coefficient. Thus in the SCOM there is the nuclear-resonant thickness parameter $\beta$ while in the CPM there is simply the parameter $N$. The relationship between the two parameters is

$$
N=\frac{\beta \Gamma}{2 f \gamma_{R}} .
$$

Equation (3) is very unusual because the left-hand side must always be an integer, while the right-hand side is certainly not, in general, an integer. The procedure for applying Eq. (2) to an actual experimental result is to evaluate the righthand side of Eq. (3) for the sample under study and then to pick $N$ as the nearest integer.

\section{COMPARISON OF THE TWO MODELS}

Before investigating the two models in detail, we will show calculations for a rather thick sample. This will bring 

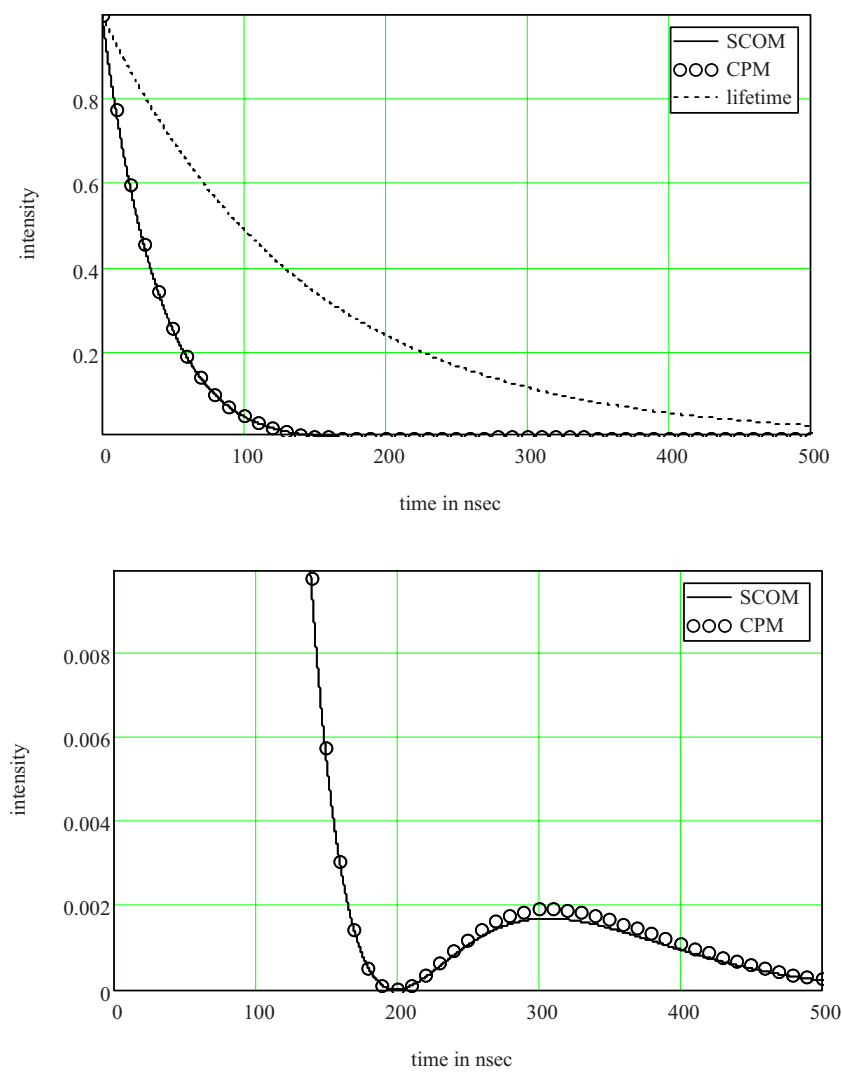

FIG. 1. (Color online) The time-dependent intensity for radiation reaching the detector assuming a rather thick sample. The two models are compared with each other and with the normal lifetime curve. The upper figure shows the speedup effect. The lower figure shows the dynamical beat effect. For this calculation the sample thickness parameter was taken to be $\beta=10.406$. This value of $\beta$ corresponds to " $N$ " $=47.764$. Thus for the CPM calculation, $N$ was set at 48 . The solid line is the SCOM result and the circles show the CPM result.

out the essential features contained in the experimental results. For all of our calculations we will use the famous Mössbauer transition in ${ }^{57} \mathrm{Fe}$ and calculate the timedependent intensity of the radiation reaching the detector. Of course the intensity is the absolute value squared of the amplitude. These results are shown in Fig. 1. Figure 1 provides the opportunity to describe the known essential features of the experimental results. These well-known features go by the names the "speedup" and "dynamical beat" effects. The speedup effect is shown in the upper portion of Fig. 1. Compare the ordinary lifetime curve with the model results. It is clear that the exponential decay rate for the thick sample is much faster than the normal lifetime. The dynamical beat effect is shown in the lower portion of Fig. 1. Notice the local maximum at a time greater than $t=0$. This is the dynamical beat. The origin of these effects is easily seen in the CPM as arising from the interference between the amplitudes of the various "paths." As remarked above, these effects have been verified experimentally. Notice further that, for this relatively thick sample, the two models give very similar results.

Now we consider the numerical comparison of the two models in some details. Kohn and Smirnov ${ }^{1}$ state that the

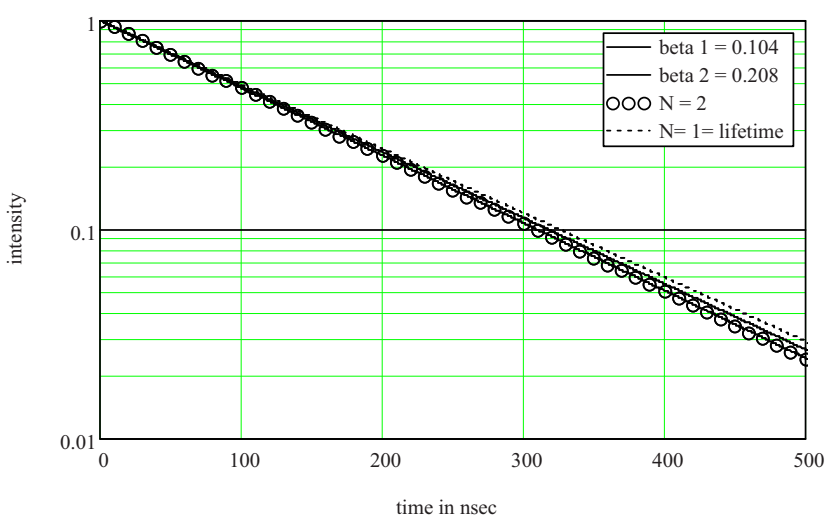

FIG. 2. (Color online) The thin sample limit is considered in this figure. In order to show any differences more clearly, a semilogarithmic plot is made. There are two curves plotted according to the SCOM corresponding to $\beta=0.104$ and $\beta=0.208$ (the solid lines). There is one curve for $N=2$ using the CPM (the circles) and the natural lifetime curve (the dotted line) is also shown. Of course for $N=1$, using the CPM, one has the natural lifetime result.

CPM "is incorrect for small sample thickness." To investigate their claim, consider Fig. 2. A semilogarithmic plot is made for Fig. 2 in order to show any differences more clearly. There are four plots in Fig. 2. There are two plots showing thin samples using the SCOM results corresponding to $\beta=0.104$ and $\beta=0.208$. These are plotted as solid lines.
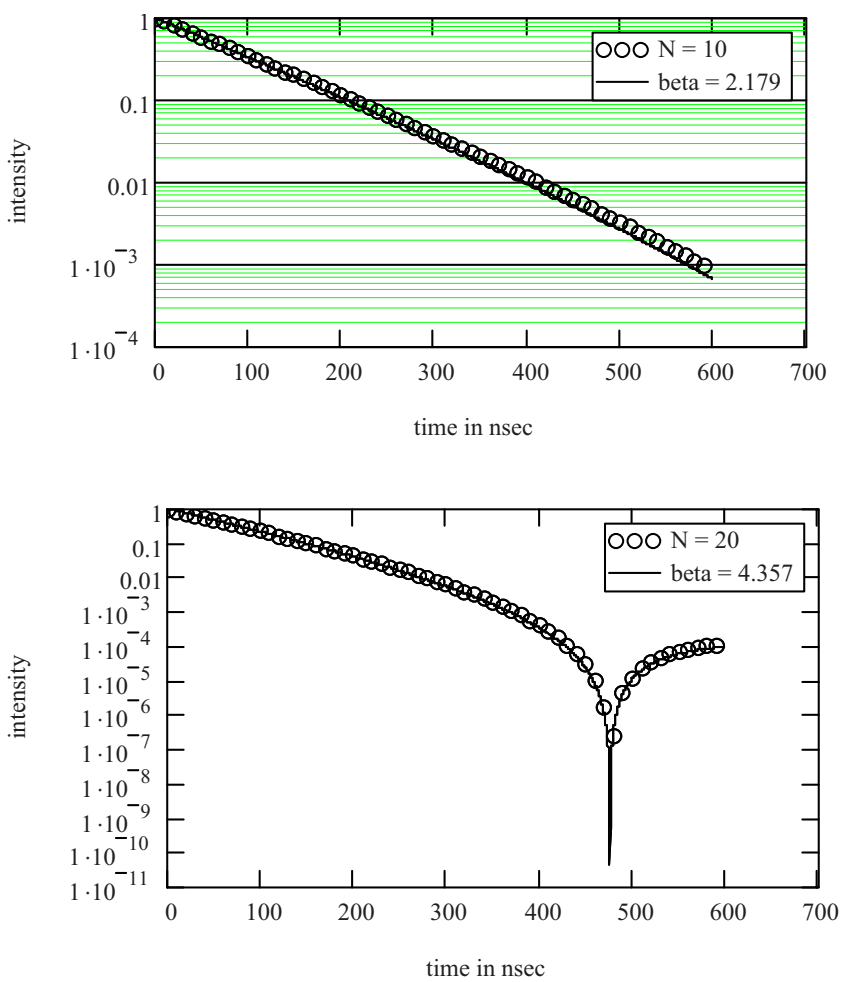

FIG. 3. (Color online) A comparison of the time-dependent intensity for radiation to reach the detector assuming the CPM and the SCOM solutions. For the upper graph the parameter for the CPM is $N=10$. The corresponding parameter for the SCOM is $\beta=2.179$. For the lower graph the parameter for the CPM is $N=20$. The corresponding parameter for the SCOM is $\beta=4.457$. 

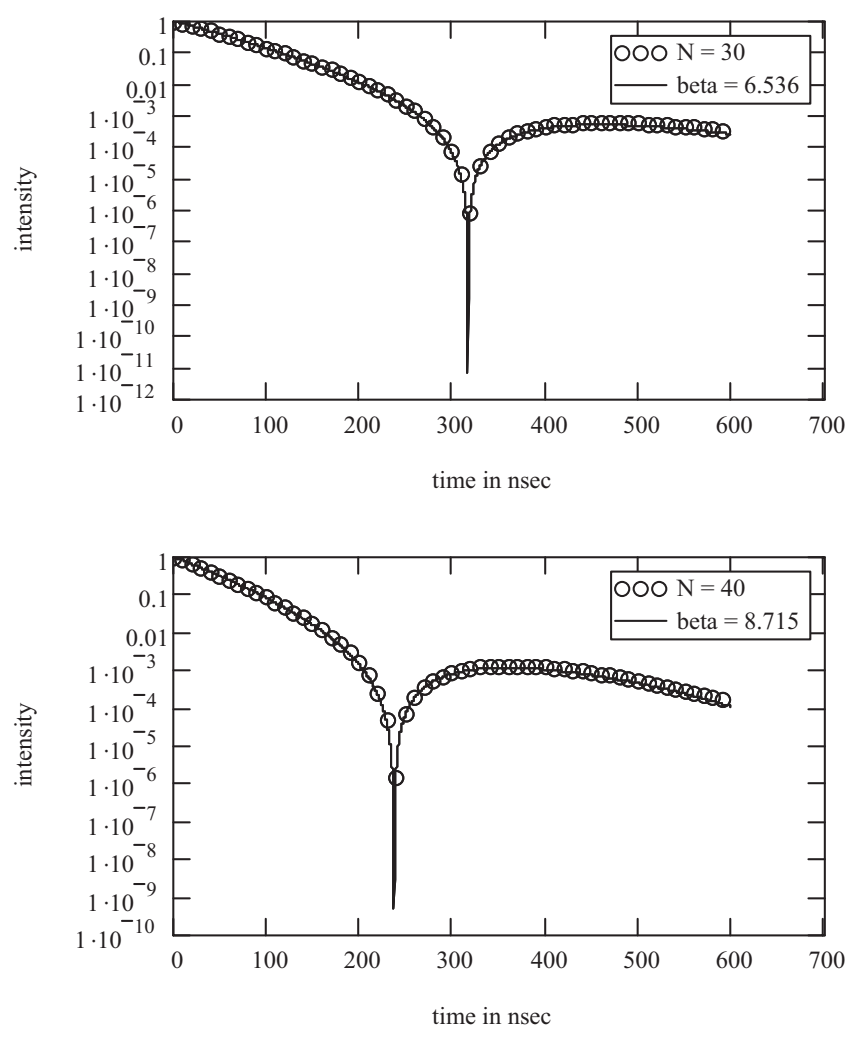

FIG. 4. A comparison of the time-dependent intensity for radiation to reach the detector assuming the CPM and the SCOM solutions. For the upper graph the parameter for the CPM is $N=30$. The corresponding parameter for the SCOM is $\beta=6.536$. For the lower graph the parameter for the CPM is $N=40$. The corresponding parameter for the SCOM is $\beta=8.715$.

There is one plot showing the natural lifetime curve, which would also correspond to the CPM result for $N=1$. This curve is given by the dotted symbol. The additional result, denoted by the circular symbols, is calculated using the CPM where $N=2$. The difference between the dotted lifetime curve and the CPM $(N=2)$ curve would allow SCOM results to be plotted for thickness values of $\beta \leq 0.208$. However, if one calculates the difference in the values of the lifetime

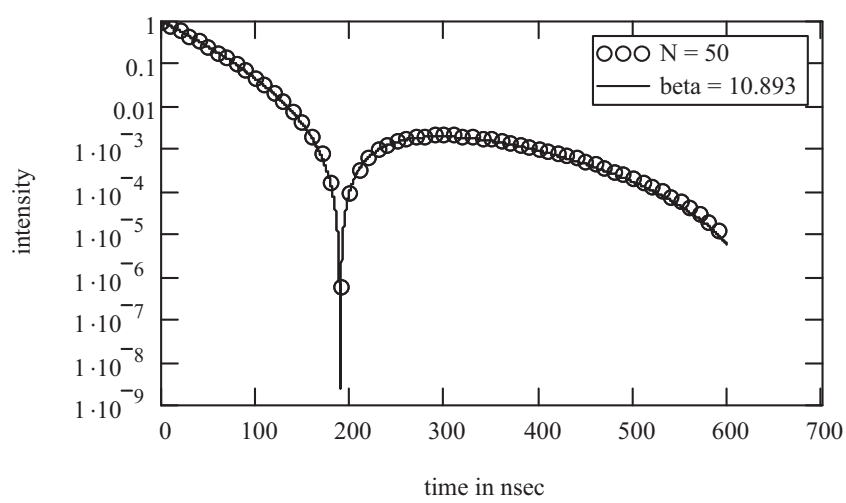

FIG. 5. A comparison of the time-dependent intensity for radiation to reach the detector assuming the CPM and the SCOM solutions. The parameter for the CPM is $N=50$. The corresponding parameter for the SCOM is $\beta=10.893$.
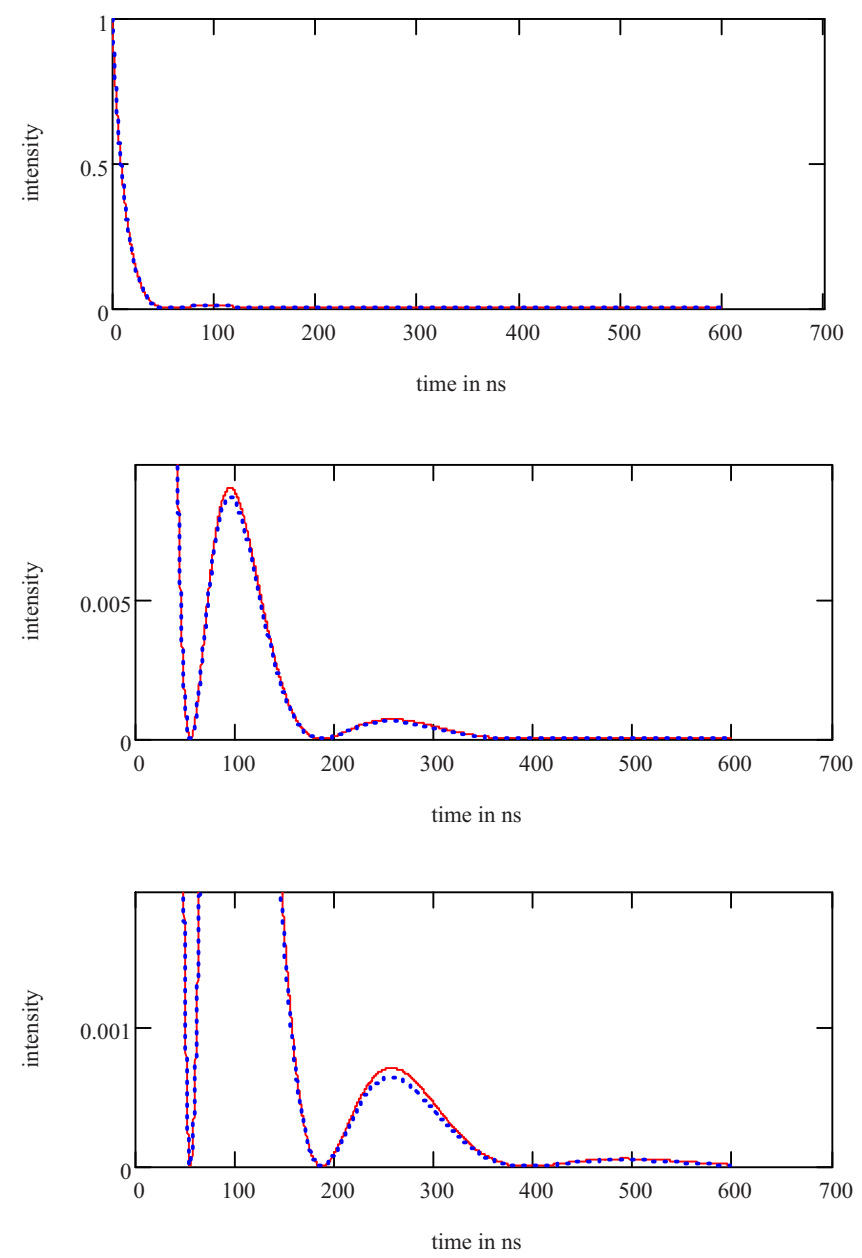

FIG. 6. (Color online) A comparison of the time-dependent intensity for radiation to reach the detector assuming the CPM and the SCOM solutions for a very thick sample. The parameter for the CPM is $N=170$. The corresponding parameter for the SCOM is $\beta$ $=37.04$.

curve and the CPM $(N=2)$ curve as a function of time, one finds the following results. The percentage difference between the two curves is $0.3 \%$ at $100 \mathrm{~ns}$ and $0.6 \%$ at $200 \mathrm{~ns}$. One sees that for thin samples, there is a range of thickness that gives essentially the same result. Thus in principle, there could be differences for small thickness but the differences would be difficult to observe. If such differences are important, time consuming experiments could be performed. This is particularly true for times up to the natural lifetime $\tau$ of 141 ns. As time increases the curves continue to diverge from each other. In an actual experiment it is very time consuming to obtain data at very late times where the counting rate is very low. Thus the conclusion is, for thin samples, both models will give essentially the same results and will agree equally well with the experimental data. Any difference between the two models can only be observed by performing very time consuming experiments.

Kohn and Smirnov ${ }^{1}$ go on to say that they can show that the difference between the two models goes to zero as $N$ $\rightarrow \infty$. In fact this issue is a bit tricky. In the first place there is essential agreement between the two theories for all thicknesses (see Figs. 3-5 for a few selected cases). Furthermore 
as $N$ goes to infinity, the intensity must go to zero, so in some sense the two theories must approach each other. The problem is to get perfect agreement for all times. In fact, what happens in the limit is that the disagreement occurs, in time, at the locations of the dynamical beat maxima and since the total intensity is very small the actual difference is very, very small (see Fig. 6).

One final numerical remark is in order. One may ask quite legitimately, due to the integer value of $N$ in the CPM, are there some samples' thicknesses that cannot be treated accurately using the CPM. Calculations show that, for all values of sample thickness, there is a difference in the two models of about $2 \%$ at time $t=100 \mathrm{~ns}$. This difference increases to about $4 \%$ at time $t=200$ ns. For all other cases, in between those shown and over larger time ranges, exactly the same trends continue as given by those shown in the figures presented.

\section{SUMMARY AND CONCLUSIONS}

The two models, namely, the coherent-path model and the semiclassical optical model can be expected to fit any experimental data concerning the time-dependent nuclear-resonant forward scattering of gamma radiation, through a resonant sample, excited by a pulsed synchrotron radiation source. Thus, if such experiments are used to obtain the nuclear parameters, both models will provide the same results. Both models show the essential features of the speedup effect, dynamical beats, and "quantum beats" when there is more than one resonant transition. It is interesting to note that the two models are of a completely different sort. The SCOM considers the nuclear-resonant sample as a continuum. The CPM treats the resonant sample as a one-dimensional chain of effective resonant nuclei. The CPM solution is in the form of a finite series for all sample thickness. Also, note that all terms in the finite series must be evaluated. The SCOM is in the form of the $J_{1}$ Bessel function. The concept of multiple scattering is completely different in the two models due to the different initial assumptions built into the models. Note also that the initial assumptions for each model are approximations to strict reality. In that regard it is worth noting the fundamental and more complete research done by Hannon and Trammell. ${ }^{8}$

It is possible that experiments could be done to determine which one of the two models gives a better fit to the data. We have no basis to presume that the coherent-path model would fit the data better than the semiclassical optical model. Moreover, it appears that such an experiment would be quite time consuming in order to obtain sufficient statistics. Since the two models give essentially the same numerical results, the value of the coherent-path model is due to the physical insight gained. It should also be noted that the CPM results ${ }^{4}$ for the radioactive source case also agree with the SCOM results ${ }^{6}$ due to Hamermesh.

Finally, one may wonder why the one-dimensional model, i.e., the CPM, provides a good description for the threedimensional case. There are a number of factors from the theory of $\mathrm{x}$-ray diffraction that may be helpful. Consider the well-known text by James. ${ }^{9}$ When the frequency of the incident radiation is comparable to the resonant frequency of the oscillating dipoles, the scattering factor will be imaginary. The imaginary part of the scattering factor denotes a component of the scattering by the dipole; the phase of which lags $\pi / 2$ behind the primary wave. Now we quote directly from James. "We have seen that the dipoles lying in any thin sheet parallel to the primary wave front produce a resultant wave whose phase is retarded $\pi / 2$ behind that of the waves scattered by the individual dipoles in the sheet. If this phase already lags $\pi / 2$ behind that of the primary wave, the retardation of the resultant scattered wave will be $\pi$, so that it opposes the primary wave." James goes on to say that this represents absorption. This analysis follows exactly the form of the coherent-path model.
${ }^{1}$ V. G. Kohn and G. V. Smirnov, Phys. Rev. B 76, 104438 (2007).

${ }^{2}$ W. Heitler, The Quantum Theory of Radiation, 3rd ed. (Oxford University Press, New York, 1957).

${ }^{3}$ S. M. Harris, Phys. Rev. 124, 1178 (1961).

${ }^{4}$ G. R. Hoy, J. Phys.: Condens. Matter 9, 8749 (1997).

${ }^{5}$ Yu. Kagan, A. M. Afanas'ev, and V. G. Kohn, J. Phys. C 12, 615 (1979).

${ }^{6}$ F. J. Lynch, R. E. Holland, and M. Hamermesh, Phys. Rev. 120,
513 (1960).

${ }^{7}$ G. R. Hoy, J. Odeurs, and R. Coussement, Phys. Rev. B 63, 184435 (2001).

${ }^{8}$ J. P. Hannon and G. T. Trammell, Hyperfine Interact. 123-124, 127 (1999)

${ }^{9} \mathrm{R}$. W. James, The Optical Principles of the Diffraction of X-Rays (Ox Bow, Woodbridge, CT, 1982), p. 138. 